\title{
QUIRI QUIRI: TOPONIMIA, MEMORIA Y GEOGRAFÍA DE LA EXACCIÓN INCA Y COLONIAL TEMPRANO EN LOS VALLES DE LONDRES Y PACINAS (SIGLOS XV-XVII)
}

\author{
QUIRI QUIRI: TOPONYMY, MEMORY AND GEOGRAPHY OF THE INCA \\ AND EARLY COLONIAL EXACTION IN THE VALLEYS OF LONDON \\ AND PACINAS (XV-XVII CENTURIES)
}

\author{
Laura Quiroga*
}

\begin{abstract}
La fuentes coloniales del siglo XVI mencionan dos provincias incas habitadas por los diaguitas al sur del Tawantinsuyu: Chicoana y Quiri Quiri. La voz Quiri Quiri desaparece tempranamente de los documentos, al tiempo que Chicoana mantiene su vigencia. Es admisible preguntarnos por qué ciertos topónimos y denominaciones colectivas persisten aún bajo el dominio colonial, en tanto otros, como Quiri Quiri, parecen caer en el olvido. Un análisis diacrónico de los enunciados textuales acerca de las provincias incas permite plantear la incidencia de un proyecto minero colonial en la región asumido como una continuidad entre el tiempo del inca y el de la conquista. La voz Quiri Quiri -de origen quechua o puquina- denomina la tierra, pero también describe el carácter de la autoridad que la gobierna: las tierras del señor áureo. Esta referencia del siglo XVI -originada con la expansión del Tawantinsuyu en los valles de los diaguitas sustenta en tiempo colonial la memoria de una región minera prehispánica, es decir, una lectura sesgada en la que solo se da cuenta de aquello que pudiera servir a la conformación y continuidad de un paisaje minero prehispánico en la nueva contingencia política colonial.
\end{abstract}

Palabras claves: Toponimia, inca, minería, diaguita, Quiri Quiri.

Colonial sources from the 16th century mention two Inca provinces in the south of Tawantinsuyu inhabited by Diaguitas: Chicoana and Quiri-Quiri. The Quiri-Quiri language disappears early from documents, while Chicoana remains. It is suitable to ask ourselves why some place names and collective denominations persist even under colonial power, meanwhile, others like Quiri-Quiri, seem to fall into oblivion. A diachronic analysis of historical texts of these Inca provinces allows us to propose the incidence of a colonial mining project in the region that can be considered as a continuum between the time of the Incas and the period of the conquest. The Quiri-Quiri language, from quechua and Puquina origins, names the land but also describes the character of the authorities that rule it: the lands of the golden god. This reference, from the 16th century, originated with the Tawantinsuyu expansion into the Diaguitas valleys, nourishes the memory of a pre-Hispanic mining region in colonial times. In other words, this reference is a biased reading that reflects what could be useful for a pre-Hispanic mining setting configuration and continuity under the new colonial policy circumstances.

Key words: Place names, inca, mining, Quiri Quiri.

\section{Introducción}

La historiografía y arqueología del antiguo Tucumán menciona la existencia de dos provincias incas al sur del Tawantinsuyu, habitadas por los diaguitas: Chicoanas y Quiri Quiri (González 1982, 1988, Raffino 2004, entre otros). En el marco de un trabajo pionero acerca de las categorías semánticas presentes en los textos coloniales, Lorandi y Busnter (1987-1988) sostienen que la voz Quiri Quiri desaparece tempranamente de los documentos, al tiempo que Chicoana mantuvo su vigencia. Es importante preguntarnos entonces por qué ciertos topónimos y denominaciones colectivas persisten a lo largo del tiempo, en tanto otros, como Quiri Quiri, objeto de nuestro trabajo, parecen caer en el olvido.

La distribución geográfica de las toponimias exhibe en forma fragmentaria y dispar los procesos históricos de una región en los que las palabras -ancladas al territorio- constituyen una forma de representación social del espacio habitado (Fernández Mier, 2006: 46). Para esto contamos con los análisis lingüísticos de toponimias en los que se entrelazan en el espacio las lenguas habladas en los Andes (Cerrón Palomino 2015; Fossa 2018). Según la interpretación del lingüista Cerrón Palomino la

* PROHAL, Programa de Historia de América Latina, Instituto de Historia Argentina y Americana Prof. E. Ravignani, Facultad de Filosofía y Letras, Universidad de Buenos Aires -CONICET. Dirección postal: Avenida Díaz Vélez 35055 D. Buenos Aires, Argentina. Correo electrónico: cotagua@yahoo.com.ar 
voz quechua quri se traduce como oro, en tanto la voz quri iqui-de origen puquina-se traduce como señor áureo (2013: 123-124).

Desconocemos los nombres que los propios diaguitas daban a los rasgos geográficos de su territorio, sin embargo, esta referencia del siglo XVI -escrita como quiri quiri-remite en tiempo colonial a la memoria de una región minera prehispánica. Basados en fuentes coloniales la historiografía ubica el asiento de Quiri Quiri en dos espacios diferentes: el valle calchaquí, cuya cabecera se encuentra en el sitio de Tolombón (Reyes Gajardo 1958; Strube 1964; Carrizo 1937; Lizondo Borda 1942) y el valle de Londres identificada con el sitio arqueológico de El Shincal (Raffino 2004) (ver Figura 1). En el caso del noroeste argentino, parte del área andina meridional, los trabajos de Alberto Rex González (1982) en las provincias del antiguo Tucumán buscaron articular la fuente histórica con la investigación arqueológica, siguiendo la metodología planteada por John Rowe para el área andina
(1982). Así se identificó el asiento de Chicoana mencionado en las fuentes, con el sitio arqueológico de la Paya. A falta de indicadores precisos en los textos, la envergadura de sus restos sirvió de base para su identificación como una supuesta cabecera.

La búsqueda de una correlación entre enclaves estatales y referencias contrastables en la palabra escrita registra dificultades metodológicas, cuando la lectura de las fuentes omite su contexto de producción. De ahí la necesidad de atender a la especificidad y estrategia metodológica propia de ambas líneas de evidencia-documentación histórica y contextos arqueológicos-, con el fin de sortear el riesgo que conlleva el uso de las analogías directas; sin embargo, el trabajo de ambos registros -escrito y material- considerados como líneas de evidencia independiente nos permite: 1 - analizar la vida material desde los textos, 2- reinterpretar las fuentes escritas a partir del análisis de las evidencias materiales y 3- reconstruir otra historia, cuando la práctica de la escritura, ligada a los intereses de
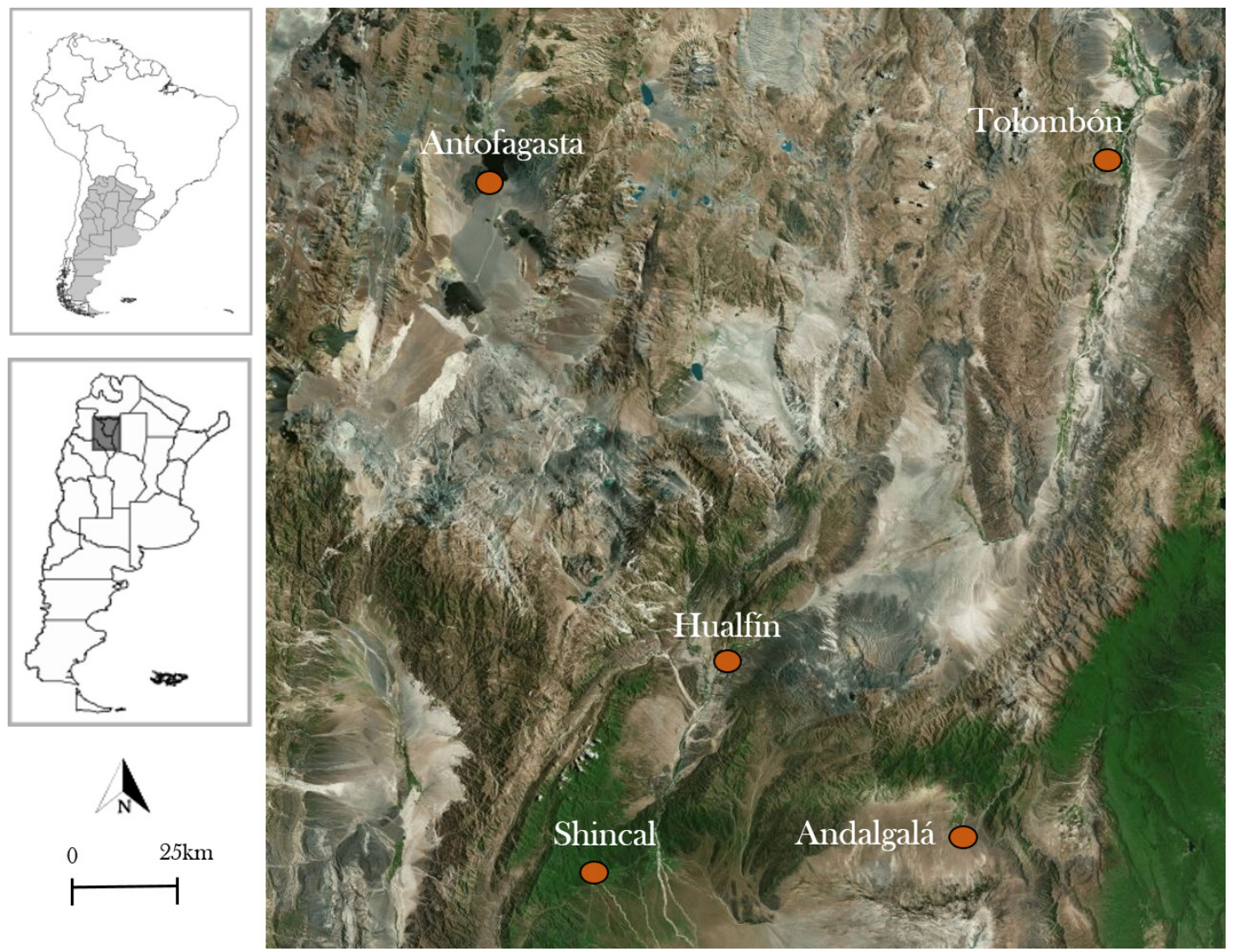

Figura 1. Ubicación del valle de Yocavil y Hualfin (Catamarca, Argentina). 
quienes detentan su control, deja en sombras o no describe ciertos aspectos de la vida social (Beaudry 1988: 1; Barceló 1988: 12 ).

Mi propuesta es que el enunciado "provincia de Quiri Quiri" en las fuentes del siglo XVI constituye una lectura colonial sesgada del ordenamiento político y económico del inca en la región, en la que solo se da cuenta de aquello que pudiera servir a la conformación de un paisaje minero - de origen prehispánico-en la nueva contingencia política. Para reconocer y dar cuenta de los sesgos coloniales que podemos rastrear en sus discursos realizamos una lectura crítica de la documentación del siglo XVI referida a la presencia inca y colonial temprano en la región. Sobre esta base confrontaremos la mirada colonial con las evidencias materiales generadas por el intenso trabajo arqueológico realizado en los valles de Londres, Hualfin y Yokavil, donde la historiografía ubica la provincia de Quiri Quiri.

Este análisis nos permite plantear tres sesgos coloniales que vamos a desplegar a lo largo de este trabajo. El primer sesgo se demuestra en la relevancia que adquiere en los textos la actividad minera y metalúrgica respecto de otras actividades, como el saber prehispánico del agua, la agricultura intensiva, los tejidos, entre otras (Lynch y Giovanetti 2018; Moralejo 2011; Tarrago et al. 2017; González 2003, entre otros). El segundo corresponde al predominio de la actividad minera aurífera mencionada en las fuentes en desmedro de la explotación y procesamiento regional del cobre mostrado por las evidencias arqueológicas (Scattolin y Williams 1992; Lynch y Giovanetti 2018). El tercero, corresponde a la envergadura de la actividad agrícola regional -incaica y de población local (Giovanetti 2015)- mencionada por los agentes coloniales solo tardíamente, en 1630, un siglo más tarde de la primera entrada conquistadora a la región cuando las rebeliones diaguitas ponen de manifiesto la envergadura y complejidad del espacio bajo cultivo y, gracias a ello, su capacidad de sostener la resistencia hasta mediados del siglo XVII (Quiroga 2011).

\section{El tiempo de los incas en la provincia de los diaguitas}

\section{Las fuentes: cronistas y probanzas}

La cronística castellana recupera la noción de provincia romana para enumerar las divisiones administrativas o regionales del Tawantinsuyu y brindar una forma de ordenar la descripción de la tierra en la que se incluye no solo el paisaje sino a sus habitantes (Martínez 2011: 13). Catherine Julien plantea que las crónicas destinadas a narrar la historia inca se basan en una forma de conciencia histórica cuzqueña expresada en una matriz narrativa genealógica. De tal forma que las genealogías de linajes reales y la sucesión de gobernantes incas vertidas por los cronistas, retoman formas de conciencia histórica cuzqueña preexistentes (Julien 2018: 64).

Es posible señalar que los relatos genealógicos también se hacen visibles en otra clase de textos, me refiero a las probanzas de méritos y servicios. Con el objeto de solicitar algún beneficio a la Corona, sobre la base de los servicios prestados por sus antepasados, el solicitante presentaba un escrito de carácter jurídico, en el que los testigos respondían un interrogatorio destinado a ratificar la declaración original (Jurado 2014).

Esta práctica, cuyos orígenes se rastrean en la Castilla medieval, se utilizó frecuentemente en los territorios americanos (Jurado 2014). La historiografía andina presenta casos en los que la elite cuzqueña y también las autoridades curacales de Charcas en tiempo colonial, apelaron a esta práctica (Espinoza Soriano 1981; Rowe 1985; Graña 2000, entre otros). Sus probanzas brindan un relato histórico habitado por genealogías, servicios y sucesos ligados a la memoria de un pasado que se actualiza, con el fin de operar en un presente marcado por nuevas autoridades y relaciones políticas. Si bien la historiografía advierte de la dudosa veracidad de las declaraciones, Jurado (2014) propone interrogar estos documentos abordando el contexto de producción de los textos jurídicos con el fin de analizarlos como herramienta de negociación política y social.

Con estas bases metodológicas vamos a presentar ambos cuerpos documentales -cronistas y probanzas- referidos a la provincia inca de Quiri Quiri o de los diaguitas, atendiendo precisamente a la distancia temporal entre los sucesos narrados y el contexto de producción del documento. De esta forma se hacen visibles los sesgos coloniales en la región bajo una memoria formada por recuerdos, olvidos y omisiones de los actores, cuando las referencias al espacio y sus habitantes constituyen herramientas políticas en una sociedad colonial en formación. 


\section{El reino de Tukma y la provincia de los diaguitas en tiempos del inca}

La cronística que relata la expansión del Tawantinsuyu mas allá de los Charcas corresponde al noveno y décimo inca, es decir Pachakuti y Tupac Inca Yupanqui, quienes alcanzan las regiones de Chile y Tucumán, pero nada nos dice de las provincias de los diaguitas. Lo cierto es que las referencias a la expansión en tiempos del décimo inca, resaltan las exploraciones mineras del gobernante hacia el sur del Cuzco en las tierras del collao, Chile y "en muchas partes" (Murúa [1615] 2019: 49v; Sarmiento de Gamboa [1572]1942: 131-132). Es probable que en ellas se incluyeran los valles de los diaguitas aunque no se mencionan de forma explícita. Entonces ¿a qué se debe esta omisión? El análisis histórico y diacrónico de las toponimias brinda algunos elementos para iniciar la búsqueda.

El Tucumán del sigo XVI, como lo definiera Lorandi, se corresponde con la gobernación creada en 1563 bajo el nombre de Tucumán, juries y diaguitas (1988, 1980). Siguiendo el planteo de Lorandi, el Tucumán se corresponde con los valles bajos y yungas del piedemonte oriental (1998: 198), en tanto Chile alcanzaría hasta las punas y valles altos trasandinos, incluyendo las tierras habitadas por diaguitas, según su interpretación de los escasos cronistas que mencionan el área. Bernabé Cobo señala que una vez derrotados los Charcas llegaron enviados del Tucumán para darle obediencia al inca:

"andando pacificando la tierra de los Charcas, le vinieron mensajeros de las remitas provincias de Tucumán, enviados de los señores dellas, que en nombre de ellos le dieron la obediencia; y el inca envio alla gobernadores y mitimaes que les enseñasen los usos y leyes de su reino" (Cobo 1653).

Las poblaciones de esta frontera oriental resguardaban los recursos que contenían los valles $\mathrm{y}$, al mismo tiempo, por medio de traslados y reasentamientos tuvieron la misión de controlar a los diaguitas serranos, quienes se mostraban rebeldes al dominio (Lorandi 1998: 203). Si la cronística deja en sombras la relación del inca con los diaguitas vamos a buscar su historia en otros textos y relatos: las genealogías contenidas en las probanzas de méritos y servicios.

\section{Acerca de la conquista de los diaguitas: las genealogías de los descendientes}

En este caso vamos a considerar tres fuentes independientes en las que se menciona la existencia de un colectivo de nombre diaguita: 1-Discurso sobre la Descendencia y Gobierno de los Incas contenida en la Biblioteca Nacional de España (BNE mss 2010) analizada por Julien (2018); 2- La probanza de méritos y servicios de los incas nietos hallada en el Archivo Histórico de Cuzco, publicada por John Rowe (1985); 3- la probanza de Juan Colque Karache, curaca de la nación killaka, ubicada en el Archivo General de Indias (AGI Charcas, 53) publicada por Espinoza Soriano (1981).

El Discurso sobre la Descendencia y Gobierno de los Incas se conoce también como Relación de los Quipucamayos. Julien sostiene que se trata de un texto escrito entre 1602 y 1608 para apoyar el reclamo de Melchor Carlos Inca a la Corona, que reproduce información provista por los quipucamayos a Vaca de Castro o Polo de Ondegardo de 1542 (2018: 74). El texto dice "Yupangue fue a quien llamaron Pachacuti Inga (...) conquistó hasta lo último de los Charcas, hasta los Chichas e Diaguitas" (BNE mss 2010, folio 52).

En segundo lugar $-\mathrm{y}$ siguiendo la perspectiva cuzqueña- contamos con la probanza de los incas nietos de conquistadores (Rowe 1985; Martínez 1995). Los descendientes de Tupa Inga Yupanqui solicitan en 1569 el reconocimiento de su ascendencia con el fin de solicitar mercedes a la Corona. La Memoria de las provincias que acompañaba el pedido de reconocimiento colonial de su linaje contenía una lista de los territorios y fortalezas conquistadas por sus antepasados: “. ...y entro en la provincia de los chichas y moyomoyos y amparais y aquitas copayapo churomatas y caracos y llego hasta los chiriguanos hasta tucuman y alli hizo una fortaleza y puso muchos indios mitimaes..." (1985: 215).

El análisis de John Rowe reconoce coincidencias entre las descripciones contenidas en la Memoria y la obra de ciertos cronistas como Sarmiento de Gamboa, Cabello Balboa y Martín de Murúa a las que denomina textos paralelos, sin embargo, para la región del Collasuyu, las comparaciones con los textos de cronistas resultan notoriamente escasas, al punto de omitir la referencia a los diaguitas en su trabajo (Rowe 1985: 215). 
Un tercer caso presentado por Juan Collque Guarache, descendiente del mallku de la nación killaka, decía que su tatarabuelo quillqi había dado la obediencia a Pachacuti y participado, bajo sus órdenes, en la conquista de chichas y diaguitas (Espinoza Soriano 1981). Este ejemplo abordado por la historiografía colonial de Charcas, muestra algunas diferencias con el caso previo, en tanto se trata de una probanza de nivel curacal que declara servicios a la autoridad del inca y luego a la Corona, reconociéndose como una autoridad intermedia sujeta y beneficiada por una autoridad superior: "si saben que el dicho Ynga Yupanqui Ynga luego como conquisto la dicha provincia fue a conquistar a las provincias de los chichas y aguitas y llevo al dicho colque por capitán general de la gente de su provincia..." (Espinoza Soriano 1981: 237).

En otras preguntas de la probanza describe su participación en la conformación del Perú colonial -como fue el enclave minero de Porco-, rol disputado con otras autoridades curacales de Charkas en cuanto a sus servicios a la Corona. En este sentido, el trabajo de Bouysse Casagne (2017) respecto de la población puquina hablante del collao en tiempos del inca propone una estrecha relación entre recursos metalíferos y sacralización del espacio. Podríamos plantear entonces que la expansión de chichas y diaguitas sería una continuidad hacia el sur de la producción minera impulsada por el inca desde el Cuzco, aspecto que aborda el trabajo de Raffino por las evidencias arqueológicas mineras en el Collao, su relación con el Cuzco y luego, en el siglo XVI, con la economía del imperio castellano (Rafiino et al. 2012).

En este trabajo nos interesa destacar solo aquellos servicios que se vinculan con el Tucumán. Me refiero a su participación y abastecimiento de la entrada de Diego de Almagro a Chile (1535), que atravesó las tierras diaguitas, y la de Diego de Rojas al Tucumán (1543). Collque Guarache declaraba méritos y servicios en una actividad sensible a los intereses de la Corona como son las exploraciones mineras y un aspecto no menor como es el conocimiento del terreno que las hacía posibles. De tal forma que si las exploraciones del inca Yupanqui de las minas al sur de los Charcas -mencionadas por Murúa- muestran la colaboración del curaca con el Tawantinsuyu, tenemos una memoria ajustada al posicionamiento político que buscaba en tiempo colonial, recuperada bajo la forma de un texto jurídico. Así se recoge el imaginario y memoria del territorio diaguita como un espacio minero del que, según sus testimonios, los Collque habían sido partícipes y habían procurado servicios imprescindibles en su conformación.

\section{La provincia inca de Quiri Quiri en el siglo XVI}

\section{Paullu inca, Diego de Almagro y los guerreros de Quiri Quiri}

En 1535 Diego de Almagro partió del Cuzco con destino a Chile, por el camino que atravesaba las tierras del Collao. La hueste reunida por Diego de Almagro llevaba consigo dos autoridades cuzqueñas: Paullu inga -hijo de Huayna Capac y hermano de Manco Inca- y Villaloma, una figura de jerarquía religiosa (Hemming 1982) ${ }^{1}$. En Tupiza el Villaloma regresa al Cuzco, en tanto Paullu continúa hacia el sur. Debido a las redes políticas que poseía en el Collao, producto de su matrimonio dentro de su propia red parental en el Titicaca, la figura de Paullu Inca tenía una especial importancia en el desarrollo de la entrada (Medinacelli 2007). Su presencia hacía posible el tránsito de la hueste por el qapap ñam garantizando así su abastecimiento, como declaran los testigos presentados en su probanza del seis de abril de 1540 (Toribio Medina, 1889, tomo V: 341-360).

Con el fin de evitar el despoblado de Atacama siguió el camino incaico que se internaba en tierras de diaguitas, ubicadas al sur de la provincia de los chichas y al oriente de los Andes. Antes de cruzar hacia Copayapo y sufrir las condiciones rigurosas de la cordillera, la probanza de Diego de Encinas de 1558 menciona de norte a sur, provincias entendidas como unidades territoriales: Xujuy, Chicuana y Quiri Quiri, o bien una sucesión de unidades culturales denominadas como naciones: charcas, chichas y diaguitas (Toribio Medina, E. 1889, Tomo VII: 207248). Para este trabajo vamos a centrarnos en los diaguitas de la provincia de Quiri Quiri, objeto de nuestro trabajo, donde la resistencia indígena adquirió la mayor escala.

$\mathrm{Al}$ sur de Chicoana, se encuentra la provincia de Quiri Quiri, sin embargo, son pocos los cronistas que consignan su nombre. Una vez que la hueste abandona Chicuana, en dirección al sur, Bartolomé Segovia -o Cristóbal de Molina- se refiere a poblados dispersos y carentes de recursos ${ }^{2}$. En el mismo 
sentido, el cronista de indias de la Corona, Gonzalo Fernández de Oviedo dedica varios capítulos en su Historia Natural de las Indias a la entrada de Diego de Almagro ${ }^{3}$. Oviedo diferencia entre los habitantes de Chiucuana y los "tres o cuatro poblecuelos de caribes" que vivían en los despoblados del sur, donde los ríos y la gente de guerra habían diezmado los caballos, ganados y recursos de la hueste en las treinta jornadas que duró la travesía, abastecidos con algarroba (Gonzalo Fernández de Oviedo 1851: 265).

Los relatos ven en Paullu una figura política y negociadora en una región que, como veremos, se muestra hostil a la presencia de la hueste. La Crónica del Reino de Chile escrita por el capitán Pedro Mariño de Lovera de 1595 en su capítulo II brinda una de las descripciones más detalladas de la provincia de Quiri Quiri ${ }^{4}$. Si bien el capitán no fue un testigo directo de los hechos que relata su versión recupera negociaciones políticas entre los líderes locales, la figura de Paulo Inca y Almagro que es pertinente considerar.

"prosiguió su camino a otra provincia llamada Quirequire. En ésta descansó algunos días, previniéndose de bastimentos, y hecha suficiente provisión fué prosiguiendo por sus jornadas hasta dar en una campaña desierta, aunque en medio de ella estaba un fuerte de dos tapias en alto, por el cual entraba un río para servicio de los que estaban dentro, que eran como quince mil indios de guerra naturales de aquella provincia, los cuales estaban bien prevenidos de mantenimientos para algunos días, y no menos de diversos géneros de armas, como gente que no atendía a cosa fuera desto". Pedro Mariño de Lovera [1595] (1865: 25).

Este párrafo sugiere la presencia de especialistas en la guerra, naturales de la región, sin embargo, en otra parte del mismo capítulo, en referencia al enfrentamiento con la hueste, afirma lo contrario: "aquella gente había concurrido de diversas provincias, cuando había llegado la voz de que venían los españoles". Pedro Mariño de Lovera [1595] (1865: 26).

La crónica se completa cuando frente a la presencia de guerreros -especialistas o no, locales o no- dispuestos a enfrentar el ingreso de la hueste, Paulo Inga busca ocupar el lugar de mediador:
"A esto respondió Pablo Inga que ya que no se quería servir dél su señoría en aquel lance en cosas de guerra, que le diese licencia para servirle en cosas concernientes a la paz, permitiendo que tratase con aquellos bárbaros sobre el negocio." Pedro Mariño de Lovera [1595] (1865: 26).

El fracaso de su gestión negociadora dio lugar a un enfrentamiento y a la derrota de los guerreros de Quiri Quiri, cuando Almagro decide finalmente enfrentarlos antes de encarar el paso de la cordillera por el puerto de la nieve, camino de Chile.

\section{Las memorias de la entrada}

Los relatos de la entrada refieren la presencia de especialistas relacionados directamente con la guerra, pero en testimonios posteriores, entre 1587 y 1589 , los testigos que guardaban la memoria de 1535 refieren a mitimaes especializados pero en la minería en las tierras de Quiri Quiri. Es claro que no habrían cambiado su actividad predominante, sino que reflejan las necesidades políticas de quienes escriben estos textos, respondiendo a un proyecto minero colonial en el Tucumán que buscaba reactivar la minería incaica y del que esperaban obtener importantes beneficios (Quiroga 2019).

Con tal motivo, entre 1587 y 1589 el gobernador Juan Ramírez de Velazco (1586-1593) recogió la memoria fragmentada de la entrada de $1535^{5}$ recopilando algunos testimonios acerca de los valles de Tulan y Çuraca "en que se dice ay mucha cantidad de indios vestidos y bien tratados y que tienen y poseen oro y plata". Dirigiendo su escrito al rey su objetivo era lograr la autorización de la Corona para realizar las exploraciones y fundaciones necesarias para hacer del Tucumán un espacio minero. Entre otros se mencionan los incas huidos de Londres o de Quiri Quiri. Los testigos entrecruzan ciertos relatos fragmentarios y dispersos entre las tierras de Lilin, Trapalanda y los cristianos del fulano César que vivían aislados, entreverados con los indios y los incas huidos de Londres que, según dijeron varios testigos, allí se habían instalado ([1587] Averiguaciones...1937: 718). El testimonio de Tula Cervin afirmaba que la tierra de Londres es muy rica de oro y plata y que esto lo había oído de Blas Ponce, partícipe de la fundación de la ciudad de Londres en 1558. En referencia a Paulo inga decía: 
"Y que en el peru rreynaban los españoles y que almagro venia aperçibido de guerra y que traiua consigo a don Pablo para la conquista de chile todos estos yngas se retiraron a lo que agora llaman çesares o linlin" ([1587] Averiguaciones...1937: 718-719).

El testimonio propio de Blas Ponce afirma que Paulo Inga había sido apresado por Almagro "para que le enseñara el camino e rriquezas". En otro fragmento agrega que en el valle de Quiri Quiri "el dicho ynga tenia sus capitanes y poblados mas de veinte mil ingas mitimaes". En el interior de su propio relato inserta el testimonio de Pedro Clavijo, quien había participado de la entrada de Diego de Rojas (1543):

"acordaron de despoblar el dicho valle de quiri quiri donde estaban por mitimaes sujetando los naturales de aquella provincia ques la que ahora llaman de Londres donde tenían sus minas y hazian sacar oro y plata" ([1587] Averiguaciones, 1937: 734)

De modo que hacia 1589 el valle de Quiri Quiri se sabía que había sido un enclave del inca, habitado entre otros por un número significativo de mitimaes destinados al control de la población local y la explotación minera.

\section{Las minas de Pacinas}

Los testimonios de soldados de la hueste que declaran sus servicios en las probanzas de méritos refieren los enclaves mineros de Gualasto y Pacinas (Quiroga 2019). En el primer caso, el topónimo conserva su vigencia hasta hoy ubicado al sur del valle de Yocavil y corroborado por evidencias arqueológicas que desarrollaremos en el apartado siguiente. Para el segundo caso, se trata de un topónimo perdido que he podido localizar en un trabajo previo (Quiroga 2019). Las minas de Pacinas, identificadas como minas del inca, se encontraban en la provincia de Quiri Quiri. La cartografía manuscrita atribuida a Ruy Díaz de Guzmán (MP-Buenos Aires, 4 del Archivo General de Indias (Sevilla, España) y las referencias brindadas por el oidor de la Audiencia de Charcas Juan de Matienzo de 1567, coinciden en ubicar Pacinas entre el valle de Londres y Calchaquí.

\begin{abstract}
"Tienese buena noticia de minas de oro, y se han visto unas minas que estan entre la provincia de Calchaqui y esta de Londres, que se llaman las de Pasinas, donde los ingas sacaban oro, y los españoles lo han sacado algunas veces". Matienzo ([1567] 1910: 191).
\end{abstract}

Ambas toponimias permanecen vigentes en el siglo XVI para sostener el proyecto minero del Tucumán, aunque luego son reemplazadas por otras referencias. Esta pérdida del topónimo nos permite plantear que Quiri Quiri y Pacinas adquieren sentido y visibilidad en el contexto de una etapa inicial de exploraciones mineras en la gobernación del Tucumán, más allá de los rendimientos que efectivamente se hubiera logrado obtener hasta mediados del siglo XVII.

\section{Los relatos materiales, la otra historia}

La lectura contextualizada de las fuentes en un marco historiográfico nos ha permitido analizar en forma crítica los textos por sí mismos, sin embargo, es posible otra lectura del proceso histórico tensionada por los contextos arqueológicos regionales. Se abre entonces un nuevo problema: cómo buscar las evidencias materiales significativas para esta parte del trabajo, en el que buscamos confrontar ambas líneas de evidencia.

Al tiempo que se construye una sacralización del espacio, la territorialidad inca plantea una geografía de la exacción mediante un sistema de caminos, enclaves y espacios productivos como instrumentos materiales de dominación, es decir, una geografía de la producción, almacenamiento y circulación del excedente (Astuhuamán Gonzáles, C. 2011.) Por esto tomamos en consideración el concepto de paisaje productivo propuesto por Lynch y Giovanetti (2018) en el que se considera la diversidad de actividades evidenciada en los sitios arqueológicos de la región. Si bien las investigaciones regionales incorporan como un rasgo central la sacralización del paisaje considero que estas formas ritualizadas de la política desplegadas por el Tawantinsuyu, están destinadas a encubrir y legitimar la apropiación del excedente por medio de una infraestructura productiva y de circulación.

Partiendo del debate historiográfico referido a la ubicación y extensión geográfica de la provincia y sus posibles cabeceras (Tolombón y El Shincal) 
podemos delimitar una región partiendo desde Tolombón (Salta, Argentina), incluyendo los valles de Yokavil, El Cajón y Andalgalá hasta los valles de Londres y Hualfin (Catamarca, Argentina) (ver Figura 1). Por otra parte, la provincia de Quiri Quiri se enuncia desde el ámbito de los valles, pero carecemos de referencias escritas directas del espacio puneño, en el que las evidencias arqueológicas no nos dejan dudas acerca de la presencia incaica en la región (Olivera y Vigliani 2000/2001).

El primer sesgo: el espacio minero. Una recopilación bibliográfica de sitios arqueológicos bajo estudio, muestra indicios inequívocos de instalaciones incaicas en la región que cartografiamos en el mapa de la Figura 2. Las referencias contienen una asignación funcional a partir de las interpretaciones brindadas por los arqueólogos que allí trabajan. En ellos se destacan varios enclaves destinados a actividades productivas agrícolas y minerometalúrgicas, estructuras de almacenamiento (los tambos) y vías de circulación, evidenciadas por el trazado del Qapaq ñan, en los que se entrecruza la funcionalidad productiva y sacralizadora del dominio del Tawantinsuyu sobre la región (Moralejo 2011, Moyano et al. 2020, Giovanetti 2015, Spina et al. 2014, Lynch 2013, Tarragó 2017, González 1997).

A lo largo del trabajo hemos puesto en evidencia que los relatos coloniales -cronistas o probanzasdescriben con insistencia un territorio minero controlado por un número indefinido de mitimaes, al tiempo que omiten la diversidad de actividades productivas que observamos a partir de la evidencia arqueológica. En este punto radica el primer sesgo colonial consistente en construir una memoria funcional a los intereses de quienes buscan en la escritura una herramienta para su posicionamiento favorable en el nuevo orden, aquel que hace de la minería su interés primordial.

El segundo sesgo: oro y cobre. Si los textos se refieren exclusivamente a las minas del inca como productoras de oro y plata, la evidencia arqueológica demuestra la importancia de la extracción y procesamiento del cobre, aspecto que las fuentes coloniales no mencionan. Tomando

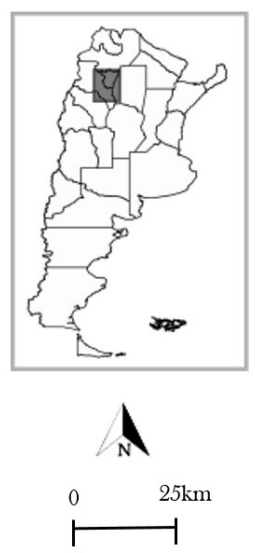

\section{Referencias}

- Enclaves agrícola

Enclaves de producción metalúrgica

Enclaves administrativos y tambos

Sitios locales con evidencias de producción metalúrgica

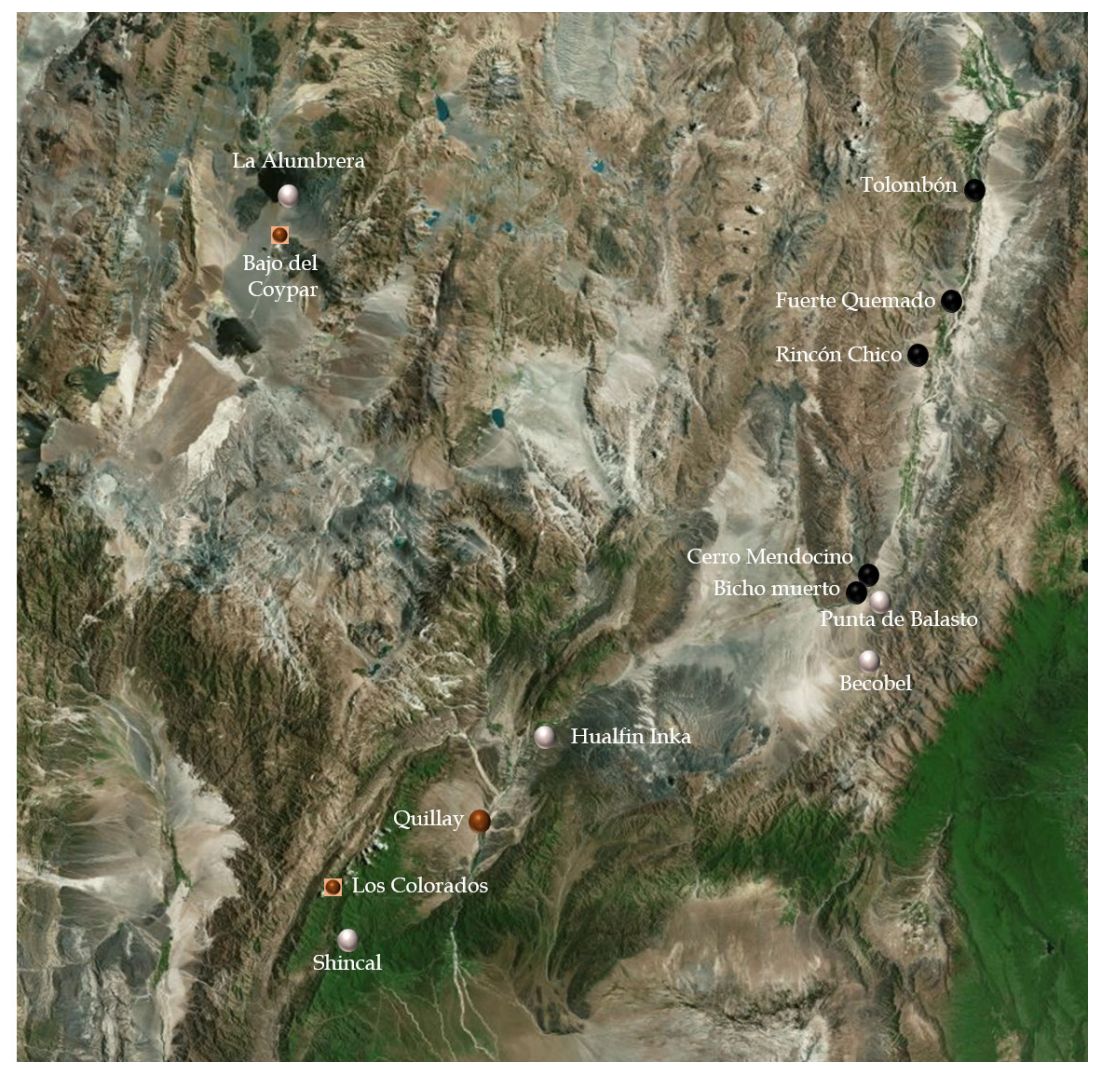

Figura 2. Sitios arqueológicos incaicos correspondientes a los valles de Yocavil y Hualfin mencionados en el texto (Catamarca, Argentina). 
en consideración la presencia de minerales como lugares de aprovisionamiento de la materia prima, observamos la distribución de sitios arqueológicos con evidencia de actividades minero-metalúrgicas del Tawantinsuyu.

El área de Hualfin y Andalgalá, donde planteamos se ubicaba el valle de Pacinas, se encuentra el sitio de Quillay (Spina et al., 2014). En el primer caso se destaca como un centro de producción por la presencia de 33 hornos de fundición del cobre obtenido en las minas de Capillitas. La ausencia de objetos terminados sugieren un proceso de producción local y su posterior traslado, siguiendo el derrotero del qapa ñan. Para el área de Andalgala se encuentra el sitio Ingenio del Arenal, ubicado al pie de las minas de Capillitas, donde la ocupación preincaica e incaica presenta evidencias de procesamiento de minerales de cobre y escasos artefactos terminados (Williams y Scattolin, 1992: 76).

El valle de Yocavil presenta evidencias de producción metalúrgica incaica en los sitios de Fuerte Quemado con la presencia de escorias y restos de moldes de bronce (Tarragó et al. 2017). Los trabajos de González respecto de las evidencias de actividades minero-metalúrgicas en el sitio Rincón Chico 15 del valle de Yocavil mencionan un aspecto central pera nuestro trabajo cuando menciona, junto con evidencias de procesamiento de cobre, el registro de moldes de lingotes asociados a evidencias de minerales de oro incrustados en los crisoles, artefactos destinados a la producción y traslado de metales preciosos hacia el Cuzco (2003: 65).

El tercer sesgo: la actividad agrícola. Las evidencias arqueológicas muestran la relevancia de la actividad agrícola regional tanto en los enclaves estatales como en los asentamientos de la población local, sea en ambiente de valles o en torno a las vegas de altura y pastizales de la Puna. La agricultura intensiva de la etapa incaica se encuentra en los enclaves productivos de Bajo del Coypar, en Antofagasta de la Sierra (Olivera y Vigliani 2000/2001). En el valle del Cajón se registra una forma arquitectónica recurrente a lo largo del Tawantinsuyu, interpretada alternativamente como espacio agrícola o de almacenamiento (de Hoyos y Williams 2017).

El paisaje productivo al norte del valle de Hualfin presenta evidencias de producción agrícola y metalúrgica (Lynch y Giovanetti 2018). El enclave de El Shincal y el sitio arqueológico Los Colorados, Giovanetti describe un sistema productivo agrícola, basado en un manejo complejo e intensivo del riego relevando la envergadura y disposición del sistema de acequias (Giovannetti 2015: 215). De tal forma que la ocupación del espacio, generación y almacenamiento del excedente agrícola cuenta con evidencias ciertas que sustentan el interés de la producción intensiva del inca en la antigua Quiri Quiri. Al mismo tiempo, las evidencias materiales ponen de manifiesto el saber prehispánico del agua de los diaguitas evidenciado en la extensión y complejidad de la base productiva de la población local de Hualfin y las laderas de Asampay (Sempé 2005: 372, Zagorodny et al. 2015).

Entonces es pertinente preguntarse cuándo y en qué contexto las descripciones coloniales hacen mención a la agricultura de los diaguitas. El saber prehispánico del manejo de aguas y cerros como espacios agrícolas adquiere visibilidad en la contingencia de la rebelión. Este sistema productivo no se menciona en las fuentes sino hasta 1630 , cuando la represión de los levantamientos indígenas en el valle de Hualfin se hace referencia a un paisaje agrícola de envergadura, sostén y garante de la población rebelde (Quiroga 2011).

\begin{abstract}
"y peleándose con el dicho enemigo en el pueblo de malfin el dicho maestre de campo tres veses en su fuerte y cordillera le mato mucha gente y ahuyentándole talándole todas sus comidas y sementeras de mais que eran capaces de mas de ochocientas fanegas de cosecha" Pedro Ramírez de Contreras, Probanza de méritos y servicios. AHC 2-6-2. Fol. 52.
\end{abstract}

\section{Conclusiones: toponimias de conquista, toponimias de la exacción}

Se plantean las conclusiones de este trabajo buscando interpretar qué proceso histórico expresan las toponimias cuando adquieren visibilidad a partir de la escritura y se confronta el texto colonial con la información de terreno. La propuesta es que los topónimos extraídos de la documentación del siglo XVI, enunciados desde el poder colonial, constituyen una red de relaciones espaciales que reinterpreta, en sus propios términos e intereses, las marcas del pasado.

Toponimias como Quiri Quiri y Pacinas se erigen como indicios de un paisaje político y productivo del inca que el sistema colonial busca apropiarse para hacer del Tucumán un espacio minero. Al mismo 
tiempo, representan la etapa de conquista, es decir, de las primeras entradas guiadas por los relatos de la hueste tras la quimera del oro. Como vimos, hacia fines del siglo XVI, cuando el gobernador Ramírez de Velazco busque emular las riquezas del Potosí, reactualiza la toponimia del inca y de la conquista que ya estaban perdiendo vigencia, merced al proceso de fundaciones urbanas y rebeliones.

La fundación de Londres de 1607, originada en los conflictos internos de la ciudad de La Rioja (1591), genera el reparto de nuevas encomiendas en la región y con ello se hacen visibles nombres colectivos como Andalgala, Famayfil, Quimivil, entre otros (Quiroga 2012). Hacia 1630, con el alzamiento general, una nueva referencia geográfica y colectiva -valle de Hualfin y malfines- emerge y se hace visible en las relaciones hispano-indígenas de la gobernación del Tucumán.

Del paisaje de conquista y de la minería incaica, expresado en los topónimos de Quiri Quiri y Pacinas, se pasa al paisaje y a las toponimias derivadas del sistema de la encomienda. En otras palabras, cuando las evidencias muestren la fragilidad del dominio, el fracaso del proyecto minero y con ello la pérdida de los topónimos que lo designaban, surgen y se hacen visibles nuevas toponimias que resultan funcionales al paisaje de la exacción colonial basada en el trabajo de los indios de encomienda.

\section{Referencias Citadas}

Astuhuamán Gonzáles, C.

2011 The concept of Inca province at Tawantinsuyu. Indiana 28: 79-107.

Averiguaciones practicadas en 1587 y 1589 por el Gobernador de Tucumán Ramírez de Velazco. Revista de la Biblioteca Nacional, Tomo I: 1, 1937: 697-747.

Barceló, M.

1988 Arqueología medieval. En las afueras del medievalismo. Critica, Barcelona.

Beaudry, M (ed.)

1988 Documentary archaeology in the New world. New Directions in Archaeology. Cambridge University Press, Cambridge.

Bouysse Casagne, $\mathrm{T}$.

2017 "Las minas de oro de los incas, el sol y las culturas del Collasuyu". Bulletin de l'ínstitut français d'etudes andines 46 (1): 9-36.

Carrizo, J. A.

1937 Cancionero popular de Tucumán. Baiocco editores, Buenos Aires.

Casanueva, F.

1993 "Crónica de una guerra sin fin: la "Crónica del reino de chile" del Capitán Pedro Mariño de Lobera (1594)". Bulletin Hispanique. Vol. 95, No 1: 119-147.

Cerrón Palomino, R.

2013 Las lenguas de los Incas: el puquina, el aimara y el quechua. PL Academic Research. Internationaler Verlag der WissenschaftenFrankfurt am Main.

Cerrón Palomino, R.

2015 "Toponimia andina: problemas y métodos". Lexis (XXXIX 1): 183-197.

de Hoyos, M., Williams, V.

2017 "Abran Kancha...: una variante de recinto perimetral compuesto en el noroeste argentino". Estudios Atacameños 55: 109-134.

Espinoza Soriano, W.

1981 "El reino aymara de Quillaca-Asanaque, siglos XVI y XVI". Revista del Museo Nacional de Lima, XLV: 175-274.
Esteve Barba, F. 1964 Historiografia indiana. Gredos. Madrid.

Fernández de Oviedo y Valdés, G.

1854 Historia General y Natural de las Indias, Islas y Tierra Firme del Mar Océano. Real Academia de la Historia, Madrid.

Fernández Mier, M.

2006 "La toponimia como fuente para la historia rural: la territorialidad de la aldea feudal". Territorio, sociedad y poder 1: $35-52$.

Fossa, L.

2018 Bajo el cielo de Chuqikirau. Editorial Horizonte, Lima. Giovannetti, M.A.

2015 Fiesta y ritos inka en el Shincal de Quimivil. Punto de Encuentro, Buenos Aires.

González, L.

2003 "Heredaras el bronce". Intersecciones en Antropología 3: $55-68$.

González, A.R.

1982 "Las provincias incas del Antiguo Tucumán". Revista del Museo Nacional. XLVI: 317-380.

Graña, Mario J.

2000 "Autoridad y memoria entre los "killakas". Las estrategias discursivas de Juan Colque Guarache en el sur andino (siglo XVI)". Histórica, Vol. 24, No 1: 23-47.

Hemming, J.

1982 La conquista de los incas. FCE, México.

Julien, C.

2018 Para leer la historia inca. Ediciones el Lector, Lima. Jurado C.

2014 "Descendentes de los primeros. Las probanzas de méritos y servicios y la genealogía cacical. Audiencia de Charcas, 1574-1719”. Revista de Indias, Vol. LXXIV, 261: 387-422.

Lamana, G.

2016 Dominación sin dominio: el encuentro inca-español en El Perú colonial temprano. Centro de Estudios Regionales Andinos Bartolomé de Las Casas, Cuzco. 
Lizondo Borda, M.

1942 Descubrimiento del Tucumán. Instituto de Historia Lingüística y Folklore. Tucumán.

Lorandi, A. M., \& Bunster, C. V.

1987-1988 "Reflexiones sobre las categorías semánticas en las fuentes del Tucumán colonial. Los valles calchaquíes". Runa, 17 (17/18): 221-262.

Lorandi, A.M.

1998 "Los diaguitas y el Tawantinsuyu: una hipótesis de conflicto". En: La frontera del estado inca, editado por Tom Dillehay y Patricia Netherly, pp. 198-215, Abya Yala, Quito.

Lynch. J.

2013 "La plaza y el Ushnu de Hualfin Inka: interpretaciones acerca de su función en la arquitectura publica del período inkaico". Boletín del Museo Chileno de Arte precolombino. (18), pp. 107-124.

Lynch, J. y Giovanetti, M.

2018 "Paisajes Inka en el centro-oeste de Catamarca (Argentina)". Latin American Antiquity, 29: 1-20.

Mariño de Lovera, Pedro

[1595] 1865 "Crónica del Reino de Chile". En: Colección de Historiadores de Chile y documentos relativos a la Historia Nacional. Santiago.

Martínez, J.L.

2011 Gente de la tierra de Guerra. Los Lipes en las tradiciones andinas y el imaginario colonial. PUCP. Lima, Perú.

Matienzo, J.

1910 [1567] Gobierno del Perú. FFyL, Sección de Historia. UBA. Buenos Aires.

Medinacelli, X.

2007 "Paullu y Manco ¿una diarquía en tiempos de conquista?". Bulletin de l'ínstitut français d'etudes andines, 36 (2): 241-258.

Moralejo, R.

2011 Los incas al sur del valle de Hualfin. Tesis doctoral. La Plata: UNLP.

Moyano, R, Moralejo, R y Couso, G.

2020 “¿Un sistema de ceques en El Shincal de Quimivil? Espacio social y arqueoastronomía en una capital incaica". Boletín del Museo Chileno de Arte Precolombino. Vol. 25, $\mathrm{N}^{\circ} 2$, 2020, pp. 165-184, Santiago de Chile.

Murúa M.

[1615] 2019 "Historia General del Piru". En: Vida y obra fray Martin de Murua. Cummins y Ossio curadores. J. Paul Getty Museum, Los Ángeles.

Olivera y Vigliani

2000/2001 "Proceso cultural, uso del espacio y producción agrícola en la Puna Meridional Argentina". Cuadernos del INAPL 19: 459-481.

Porras Barrenechea, R.

1943 Epílogo crítico-bibliográfico. Las crónicas de los Molinas. Asociación editora. Serie I, Tomo IV: 85-98, Lima.

Quiroga, L.

2011 "Al Abrigo de sus Huaycos: Narrar la geografía, habitar los espacios, interpretar las prácticas”. En: María E. Albeck, Beatriz Cremonte y Marta Ruiz eds. Sociedades Precolombinas Surandinas. TANOA II. Universidad de Jujuy.

Quiroga, L.

2012 "Las granjerías de la tierra: Actores y escenarios del conflicto colonial en el valle de Londres (Gobernación del
Tucumán) (1607-1611)”. Surandino Monográfico. 2. Revista electrónica del Programa de Historia de América Latina (PROHAL).

Quiroga, L.

2019 "Las minas del inca y el valle de Pasinas (Siglo XV-XVI, Gobernación del Tucumán, Virreinato del Perú)". Revista Intercambios. Estudios de Historia y Etnohistoria. Escuela Nacional de Antropología e Historia. No 4: 43-50. México.

Raffino, R.

2004 El Shincal de Quimivil. Sarquis, Catamarca.

Raffino, R. Gobbo, J. Iacona A, Moralejo, R.

2012 "La minería y metalurgia de los Inkas del Kollasuyu". En: Actas del V Congreso Nacional de Arqueología Histórica Tomo 1: 187-216. Editorial Académica española.

Reyes Gajardo

1958 "Poblaciones indígenas del Valle Calchaquí". UNT Revista del Instituto de Antropología, Vol. VIII. 24-59.

Rowe, J.

1982 "Inca policies and institutions relating to the cultural unification of the empire". En The Inca and Aztec states, 1400-1800; anthropology and history, edited by George A. Collier, Renato I. Rosaldo, John D. Wirth, pp. 93-118. Academic Press, New York, Londres.

Rowe, J.

1985 "Probanza de los incas nietos de conquistadores". Histórica. Vol. IX, № 2: 193-245.

Sarmiento de Gamboa, P.

[1573] 1942 Historia de los Incas. Segunda edición. Colección Horreo. Emece Editores. Buenos Aires.

Sempé, M.C.

2005 Azampay: Pasado y presente de un pueblito catamarqueño. Sempé C. Salceda, S. Maffia. M. compiladoras. Ediciones al Margen, La Plata.

Spina, J., Giovanetti, M. y Ferraris, E.

2014 "Interrogantes de la prehispánica andina. Nuevas propuestas desde Los Hornos de Quillay (Catamarca, Argentina)". Chungara (49) 3: 327-342.

Strube E.

1963 "La Ruta de D. Diego de Almagro a Chile: Preliminares". Revista de Historia de América. No 55/56: 1-26.

Scattolin, M.C., Williams, V.

1992 "Actividades minero-metalúrgicas prehispánicas en el Noroeste argentino. Nuevas evidencias y su significación". Bulletin de l'Institut Français d'Etudes Andines (21): 59-87.

Spina, J., Giovanetti, M. y Ferraris, E.

2014 "Interrogantes de la prehispánica andina. Nuevas propuestas desde Los Hornos de Quillay (Catamarca, Argentina)". Chungara, Revista de Antropología Chilena (49) 3: 327-342.

Tarrago, M., Marchegiani, M., Palamarczuk, V., Reynoso, A. 2017 "Presencia del inca en Yocavil (Catamarca, Argentina). Integración en la diversidad". Boletín del Museo Chileno de Arte Precolombino, 22, № 1: 95-117.

Toribio Medina, J.

1889 Colección de Documentos inéditos para la Historia de Chile desde el viaje de Magallanes hasta la batalla del Maipo. 1518-1818. Imprenta Ercilla, Santiago de Chile.

Zagorodny, N., Angiorama, Becerra Perez Pieroni

2015 "Evidenicas de actividades metalúrgicas en el sitio Campo Carrizal (Belén, Catamarca)". Intersecciones de Antropología (16): 439-450. 


\section{Notas}

1 La presencia de ambas figuras se explica desde diferentes estrategias políticas de las relaciones hispano-incaicas en los primeros años. Como una forma de desplazar el potencial político de Paulo en desmedro de Manco Inca (Hemming 1982), como encargado de desplazar la figura de Almagro de los intereses de los pizarristas en Cuzco (Lamana 2016) o como componente de una diarquía que fluctúa entre la negociación y la resistencia (Medinacelli 2007).

2 Este documento consultado en el portal de archivos españoles (pares.es) con la signatura AGI, Patronato 8, R.12 carece de firma y fecha en el original digitalizado. Porras Barrenechea (1943: 91) la atribuye al clérigo Bartolomé Segovia, participante de la entrada.

3 Esta obra describe los hechos entre 1535 hasta 1548. La tercera parte donde se narran los hechos de la entrada permanecieron inéditos hasta 1851 , cuando fue editada por la Academia de la Historia de España, edición utilizada en este trabajo (Esteve Barba 1964: 71).

4 Pedro Mariño de Lobera fue un soldado español que participó en la guerra de Arauco. La obra relata los hechos sucedidos en el Reino de Chile entre 1535 y 1595 . Su texto fue revisado y corregido por el jesuita Bartolomé de Escobar, quien destaca su rol de testigo o de conocedor indirecto de los sucesos que narra, los que "inquirio con tanta solicitud" (Casanueva 1993: 119)

5 "Ynformacion que çerca de la ciudad de Cordoba de la gobernación de tucuman ay 70 o 80 leguas esta una provincia que se llama el valle de talan y çuraca en que se dice ay indios vestidos y bien tratados y tienen oro y plata". 